11

\title{
Эффективность обнаружения одного класса сверхширокополосных сигналов в условиях параметрической априорной неопределенности
}

\author{
(С) А.П. Трифонов, Ю.Э. Корчагин, К.Д. Титов
}

Воронежский государственный университет,

394018 Воронеж, Россия

ฯ e-mail: korchagin@phys.vsu.ru

(Поступило в Редакцию 9 ноября 2017 г.)

Синтезирован квазиправдоподобный алгоритм обнаружения сверхширокополосного квазирадиосигнала произвольной формы с неизвестными амплитудой, начальной фазой и длительностью. Найдены статистические характеристики эффективности функционирования синтезированного алгоритма обнаружения вероятности ложной тревоги и пропуска сигнала. Определены потери в эффективности обнаружения вследствие априорного незнания параметров сигнала.

DOI: $10.21883 / J T F .2018 .08 .46315 .2552$

В современных практических приложениях радио- и гидролокации, навигации, сейсмологии, радиосвязи и др. все более широкое применение находят сверхширокополосные сигналы (СШП, UWB) [1-3]. При этом, как правило, используется амплитудно-импульсная и времяимпульсная модуляция, что приводит к необходимости приема СШП сигнала с неизвестными амплитудой и временем прихода. Оптимальные и квазиоптимальные алгоритмы обработки СШП сигналов с неизвестными амплитудой и временем прихода исследованы, например, в $[4,5]$. Однако в силу особенностей процесса распространения, а также из-за возможной неточности модели на приемной стороне часто оказывается неизвестной длительность СШП сигнала. Задача обнаружения сигнала с неизвестной длительностью актуальна не только для СШП сигналов, она неоднократно рассматривалась в литературе применительно к узкополосным радиосигналам [6-8]. В случае неизвестных длительности, амплитуды и начальной фазы радиосигнала синтез обнаружителя связан с необходимостью преодоления параметрической априорной неопределенности. Были исследованы квазиправдоподобный (КП) и максимально правдоподобный (МП) алгоритмы обнаружения узкополосного радиосигнала. В первом из них вместо неизвестных параметров используются некоторые их ожидаемые значения, а во втором - МП оценки неизвестных параметров [6-8]. Однако результаты решения задач обнаружения узкополосных радиосигналов неприменимы к СШП сигналам, поскольку при получении известных результатов существенно использовалось условие относительной узкополосности. Поэтому представляет интерес синтез и анализ алгоритмов обнаружения СШП сигналов с неизвестной длительностью. Класс СШП сигналов чрезвычайно широк и включает в себя много различных моделей. Среди СШП выделим подкласс таких сигналов, структура которых подобна узкополосным радиосигналам, однако условие относительной узкополосности для них не выполняется. Такие сигналы в работе [1] названы СШП квазирадиосигналами (КРС). В рамках этой терминологии узкополосные радиосигналы являются частным случаем СШП КРС. Поэтому довольно актуальной представляется задача обобщения результатов решения задач обнаружения узкополосных радиосигналов на класс СШП КРС. Следует также заметить, что само понятие относительной узкополосности является в классической радиофизике, скорее, качественным, чем количественным. Решение задач обнаружения СШП КРС позволяет в каждом конкретном случае сформулировать количественные критерии узкополосности.

Модель сигнала, подлежащего обнаружению, запишем в виде

$$
s(t, a, \varphi, \tau)= \begin{cases}a f(t) \cos (\omega t-\varphi), & 0 \leq t \leq \tau, \\ 0, & t<0, t>\tau,\end{cases}
$$

где $a, \varphi, \omega, \tau$ - его амплитуда, начальная фаза, частота и длительность соответственно, а $f(t)$ - модулирующая функция. Если полоса частот $\Delta \omega$ и центральная частота $\omega$ удовлетворяют условию

$$
\Delta \omega \ll \omega,
$$

то сигнал (1) является узкополосным радиосигналом $[1,7]$. Если условие (2) не выполняется, то формула (1) описывает СШП КРС [1,4,5].

Блок-схема формирования сигнала (1) изображена на рис. 1, где обозначено: $G$ - генератор гармонического

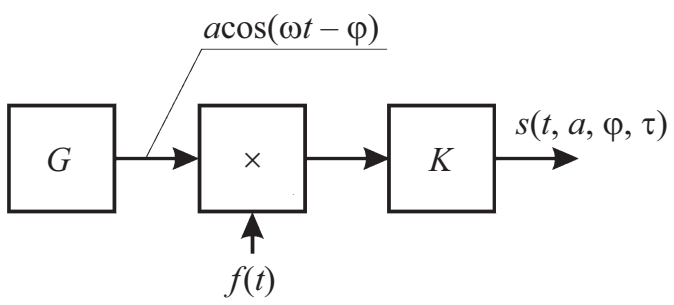

Рис. 1. Блок-схема формирования СШП КРС. 
сигнала $a \cos (\omega t-\varphi), K-$ ключ, замкнутый на интервале времени $[0, \tau]$. Величины $a, \varphi$, и $\omega$ являются параметрами гармонического колебания, используемого для формирования СШП КРС. Тем не менее аналогично $[1,4,5]$ далее будем называть $a, \varphi, \omega$ соответственно амплитудой, начальной фазой и частотой СШП КРС (1). Выбором модулирующей функции $f(t)$ можно обеспечить полосу $\Delta \omega$ сигнала, близкую к частоте $\omega$. Таким образом, изменение модулирующей функции $f(t)$ позволяет описать формулой (1) как СШП КРС с большой относительной полосой частот, так и узкополосные радиосигналы, для которых выполняется условие (2) [1,4,5].

Будем полагать, что сигнал (1) принимается на фоне белого гауссовского шума $n(t)$ с односторонней спектральной плотностью $N_{0}$, а истинные значения амплитуды $a_{0}$, начальной фазы $\varphi_{0}$ и длительности $\tau_{0}$ a priori неизвестны. Наблюдаемую в течение интервала времени $t \in[0, T]$ аддитивную смесь сигнала (1) и шума $n(t)$ представим в виде

$$
\xi(t)=\gamma_{0} s\left(t, a_{0}, \varphi_{0}, \tau_{0}\right)+n(t),
$$

где $\gamma_{0}-$ неизвестное истинное значение дискретного параметра $\gamma$, принимающего значение $\gamma=0$ при отсутствии сигнала и $\gamma=1$ - при его наличии. Будем считать, что длительность сигнала может принимать значения из априорного интервала $\tau \in\left[T_{1}, T_{2}\right]$, $0<T_{1}<T_{2} \leq T$. Располагая принятой реализацией (3), приемное устройство должно выносить решение о наличии или отсутствии сигнала. Следовательно, задача обнаружения сводится к оценке дискретного параметра $\gamma$ на основе наблюдаемых данных (3).

Для синтеза алгоритма обнаружения СШП КРС (оценки параметра $\gamma$ ) воспользуемся методом МП $[6,7,9]$. При неизвестных параметрах сигнала имеет место априорная параметрическая неопределенность относительно амплитуды, начальной фазы и длительности. В этом случае логарифм функционала отношения правдоподобия (ФОП) зависит от четырех неизвестных параметров

$$
\begin{aligned}
L(\gamma, a, \varphi, \tau)= & \frac{2 \gamma}{N_{0}} \int_{0}^{T} \xi(t) s(t, a, \varphi, \tau) d t \\
& -\frac{\gamma}{N_{0}} \int_{0}^{T} s^{2}(t, a, \varphi, \tau) d t .
\end{aligned}
$$

Ряд алгоритмов обнаружения может быть получен при подстановке в выражение (4) вместо неизвестных $a, \varphi$ и $\tau$ их некоторых значений. Эти значения могут быть фиксированными, а могут определяться по реализации наблюдаемых данных. В работе [10] исследован КП алгоритм обнаружения СШП КРС с неизвестными амплитудой, начальной фазой и длительностью, где вместо a priori неизвестной длительности использовано некоторое ее ожидаемое (прогнозируемое) значение, а по неизвестным амплитуде и начальной фазе выполнялась адаптация. В настоящей работе рассмотрен КП алгоритм обнаружения СШП КРС с адаптацией по длительности. Вместо неизвестных амплитуды и начальной фазы в выражении (4) будем аналогично [10] использовать некоторые их ожидаемые значения $a^{*}$ и $\varphi^{*}$ соответственно, а вместо неизвестной длительности - ее КП оценку (что равносильно адаптации алгоритма обнаружения по длительности). Тогда КП оценка $\hat{\gamma}$ параметра $\gamma$, определяется как значение $\gamma$, при котором логарифм ФОП достигает абсолютного (наибольшего) максимума [9], т.е. КП алгоритм обнаружения сигнала (оценки параметра $\gamma$ ) можно аналогично [10] представить в виде

$$
\hat{\gamma}= \begin{cases}1, & L \geq h, \\ 0, & L<h .\end{cases}
$$

Здесь

$$
L=\sup _{\tau} L(\tau), \quad L(\tau)=L\left(\gamma=1, a=a^{*}, \varphi=\varphi^{*}, \tau\right) .
$$

Порог $h$ в формуле (5) выбирается в соответствии с заданным критерием оптимальности [6,7]. Выражения (4)-(6) определяют структуру приемного устройства. Обнаружитель должен формировать случайный процесс (6) для всех возможных значений длительности и находить величину его максимума. Решение о наличии или отсутствии сигнала выносится на основе сравнения величины максимума (6) с порогом $h$. Подставим в выражение (4) явный вид СШП КРС (1) и преобразуем логарифм ФОП к виду

$$
\begin{aligned}
& L(\tau)=a^{*}\left(X(\tau) \cos \varphi^{*}+Y(\tau) \sin \varphi^{*}\right) \\
& -a^{* 2}\left[Q(\tau)+P_{c}(\tau) \cos 2 \varphi^{*}+P_{s}(\tau) \sin 2 \varphi^{*}\right] / 2,
\end{aligned}
$$

где обозначено

$$
\begin{gathered}
X(\tau)=\frac{2}{N_{0}} \int_{0}^{\tau} \xi(t) f(t) \cos \omega t d t, \\
Y(\tau)=\frac{2}{N_{0}} \int_{0}^{\tau} \xi(t) f(t) \sin \omega t d t, \\
Q(\tau)=\frac{1}{N_{0}} \int_{0}^{\tau} f^{2}(t) d t, \\
P_{c}(\tau)=\frac{1}{N_{0}} \int_{0}^{\tau} f^{2}(t) \cos (2 \omega t) d t, \\
P_{S}(\tau)=\frac{1}{N_{0}} \int_{0}^{\tau} f^{2}(t) \sin (2 \omega t) d t .
\end{gathered}
$$

КП обнаружитель СШП КРС (5) можно реализовать на основе блок-схемы, приведенной на рис. 2, где 


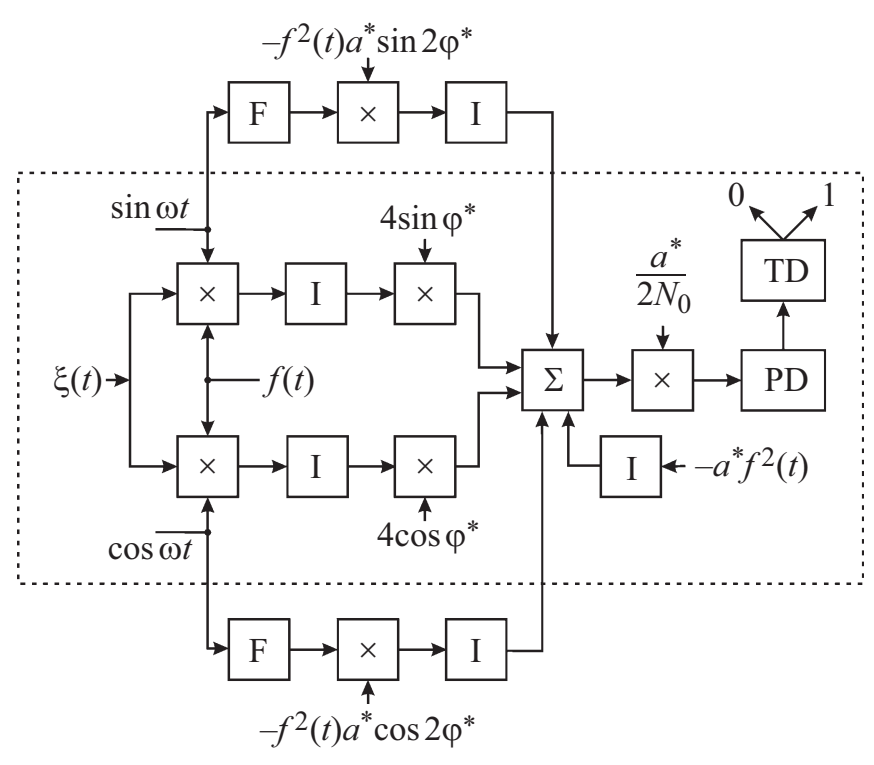

Pис. 2. Блок-схема КП обнаружителя СШП КРС.

интеграторы (I) работают на интервале времени $[0, t]$, $t \in\left[0, T_{2}\right], F-$ удвоители частоты, PD - пиковый детектор, TD - пороговое устройство, которое осуществляет сравнение величины максимума $L$ с порогом $h$ и выносит решение о наличии или отсутствии сигнала. Для узкополосного радиосигнала $\left|P_{c}(\tau)\right| \ll Q(\tau)$, $\left|P_{s}(\tau)\right| \ll Q(\tau)$ и логарифм ФОП (7) приближенно можно записать в виде

$$
\begin{aligned}
L(\tau) \approx L_{n}(\tau)= & a^{*}\left(X(\tau) \cos \varphi^{*}+Y(\tau) \sin \varphi^{*}\right) \\
& -a^{* 2} Q(\tau) / 2 .
\end{aligned}
$$

Блок-схема КП обнаружителя узкополосного радиосигнала обведена на рис. 2 штриховой линией. Как видно из рисунка, учет возможной неузкополосности принимаемого сигнала приводит к усложнению блок-схемы обнаружителя, в частности, к необходимости использования удвоителей частоты и перемножителей.

Выполним анализ КП алгоритма обнаружения (5), т.е. найдем вероятности ложной тревоги и пропуска сигнала $[6,7,11]$. Очевидно, что незнание амплитуды и начальной фазы влияет на эффективность обнаружения. Поэтому введем в рассмотрение величины, характеризующие расстройку КП обнаружителя по амплитуде $\Delta_{a}=a^{*} / a_{0}$ и начальной фазе $\Delta_{\varphi}=\varphi^{*}-\varphi_{0}$. Тогда ожидаемые амплитуду и начальную фазу можно выразить через истинные значения и расстройки как $a^{*}=a_{0} \Delta_{a}$ и $\varphi^{*}=\varphi_{0}+\Delta_{\varphi}$. Подставив ожидаемые значения $a^{*}$ и $\varphi^{*}$ в выражение (7), запишем логарифм ФОП в виде

$$
\begin{aligned}
L(\tau)= & a_{0} \Delta_{a}\left(X(\tau) \cos \left(\varphi_{0}+\Delta_{\varphi}\right)+Y(\tau) \sin \left(\varphi_{0}+\Delta_{\varphi}\right)\right) \\
& -\frac{\left(a_{0} \Delta_{a}\right)^{2}}{2}\left(Q(\tau)+P_{c}(\tau) \cos \left(2 \varphi_{0}+2 \Delta_{\varphi}\right)\right. \\
& \left.+P_{s}(\tau) \sin \left(2 \varphi_{0}+2 \Delta_{\varphi}\right)\right) .
\end{aligned}
$$

Случайные процессы $X(\tau)$ и $Y(\tau)$ являются гауссовскими, поскольку представляют собой линейные преобразования (8) гауссовского случайного процесса (3), следовательно, случайный процесс (9) также является гауссовским. Поэтому для его полного статистического описания достаточно найти математическое ожидание и корреляционную функцию. Обозначим $L_{1}(\tau)=\left\{L(\tau) \mid \gamma_{0}=1\right\}$ - логарифм ФОП (9) при наличии сигнала в принятой реализации, а $L_{0}(\tau)=\left\{L(\tau) \mid \gamma_{0}=0\right\}-$ при его отсутствии. Выполняя усреднение, получаем математические ожидания при наличии сигнала

$$
\begin{aligned}
S_{1}(\tau)= & \left\langle L_{1}(\tau)\right\rangle=a_{0}^{2} \Delta_{a}\left[Q\left(\min \left(\tau, \tau_{0}\right)\right) \cos \left(\Delta_{\varphi}\right)\right. \\
& +P_{c}\left(\min \left(\tau, \tau_{0}\right)\right) \cos \left(2 \varphi_{0}+\Delta_{\varphi}\right) \\
& \left.+P_{s}\left(\min \left(\tau, \tau_{0}\right)\right) \sin \left(2 \varphi_{0}+\Delta_{\varphi}\right)\right] \\
& -\frac{a_{0}^{2} \Delta_{a}^{2}}{2}\left[Q(\tau)+P_{c}(\tau) \cos \left(2 \varphi_{0}+2 \Delta_{\varphi}\right)\right. \\
& \left.+P_{s}(\tau) \sin \left(2 \varphi_{0}+2 \Delta_{\varphi}\right)\right]
\end{aligned}
$$

и при его отсутствии

$$
\begin{aligned}
S_{0}(\tau)= & \left\langle L_{0}(\tau)\right\rangle=-\frac{a_{0}^{2} \Delta_{a}^{2}}{2}\left[Q(\tau)+P_{c}(\tau) \cos \left(2 \varphi_{0}+2 \Delta_{\varphi}\right)\right. \\
& \left.+P_{s}(\tau) \sin \left(2 \varphi_{0}+2 \Delta_{\varphi}\right)\right]
\end{aligned}
$$

а также корреляционную функцию

$$
\begin{aligned}
& K\left(\tau_{1}, \tau_{2}\right)=\left\langle\left[L_{1}\left(\tau_{1}\right)-\left\langle L_{1}\left(\tau_{1}\right)\right\rangle\right]\left[L_{1}\left(\tau_{2}\right)-\left\langle L_{1}\left(\tau_{2}\right)\right\rangle\right]\right\rangle \\
& \quad=\left\langle\left[L_{0}\left(\tau_{1}\right)-\left\langle L_{0}\left(\tau_{1}\right)\right\rangle\right]\left[L_{0}\left(\tau_{2}\right)-\left\langle L_{0}\left(\tau_{2}\right)\right\rangle\right]\right\rangle \\
& \quad=a_{0}^{2} \Delta_{a}^{2}\left[Q\left(\min \left(\tau_{1}, \tau_{2}\right)\right)+P_{c}\left(\min \left(\tau_{1}, \tau_{2}\right)\right)\right. \\
& \left.\quad \times \cos \left(2 \varphi_{0}+2 \Delta_{\varphi}\right)+P_{s}\left(\min \left(\tau_{1}, \tau_{2}\right)\right) \sin \left(2 \varphi_{0}+2 \Delta_{\varphi}\right)\right] .
\end{aligned}
$$

Далее будем полагать, что выходное отношение сигнал-шум (ОСШ) для принятого сигнала достаточно велико. Для нахождения вероятности ложной тревоги исследуем решающую статистику $L_{0}(\tau)$ в окрестности положения ее максимума. С увеличением ОСШ положение максимума решающей статистики сходится в среднеквадратическом к положению максимума ее математического ожидания [11]. Производная математического ожидания (10) при отсутствии сигнала

$$
\frac{\partial S_{0}(\tau)}{\partial \tau}=-a_{0}^{2} \Delta_{a}^{2} f^{2}(\tau) \cos ^{2}\left(\omega \tau-\varphi_{0}-\Delta_{\varphi}\right) / N_{0}
$$

при всех возможных значениях длительности отрицательна. Следовательно, положение максимума математического ожидания $S_{0}(\tau)$ решающей статистики на отрезке $\left[T_{1}, T_{2}\right]$ совпадает с левой границей априорного интервала возможных значений длительности $T_{1}$. Разложив выражения (11) и (12) в ряды Тейлора по $\tau$ в окрестности $T_{1}$, получим асимптотические выражения 
для математического ожидания и корреляционной функции при отсутствии сигнала

$$
\begin{gathered}
S_{0}(\tau) \approx-\lambda_{0} / 2-\left(\tau-T_{1}\right) \psi_{0} / 2 T_{2}, \\
K_{q 0}\left(\tau_{1}, \tau_{2}\right) \approx \lambda_{0}+\psi_{0} \min \left(\tau_{1}-T_{1}, \tau_{2}-T_{1}\right) / T_{2},
\end{gathered}
$$

где

$$
\begin{gathered}
\psi_{0}=\Delta_{a}^{2} z^{2} f^{2}\left(T_{1}\right) \cos ^{2}\left(\omega T_{1}-\varphi_{0}-\Delta_{\varphi}\right), \\
\lambda_{0}=\left(a_{0} \Delta_{a}\right)^{2}\left(Q\left(T_{1}\right)+P_{c}\left(T_{1}\right) \cos \left(2 \varphi_{0}+2 \Delta_{\varphi}\right)\right. \\
\left.+P_{s}\left(T_{1}\right) \sin \left(2 \varphi_{0}+2 \Delta_{\varphi}\right)\right), \quad z^{2}=2 a_{0}^{2} T_{2} / N_{0}
\end{gathered}
$$

- ОСШ на выходе приемника МП для СШП КРС с амплитудой $a_{0}$ и длительностью $T_{2}$ и прямоугольной модулирующей функцией.

Будем аппроксимировать логарифм ФОП $L_{0}(\tau)$ при больших ОСШ гауссовским случайным процессом $\mu_{0}(\tau)$ с математическим ожиданием (13) и корреляционной функцией (14) на всем априорном интервале значений длительности. Используя выражения (13), (14) и теорему Дуба $[12,13]$, можно показать, что решающая статистика $\mu_{0}(\tau)$ является гауссовским марковским процессом с коэффициентом сноса $k_{10}$ и коэффициентом диффузии $k_{20}[12,13]$

$$
k_{10}=-\psi_{0} / 2 T_{2}, \quad k_{20}=\psi_{0} / T_{2} .
$$

Вероятность ложной тревоги по определению равна $\alpha=1-F_{0}(h)$, где

$$
F_{0}(h)=P\left\{\mu_{0}(\tau)<h, \quad \tau \in\left[T_{1}, T_{2}\right]\right\}
$$

- вероятность недостижения границ $y=-\infty$ и $y=h$ марковским случайным процессом $\mu_{0}(\tau)$ на интервале $\tau \in\left[T_{1}, T_{2}\right]$. Искомую вероятность (17) можно выразить через плотность вероятности $W(y, \tau)$ реализаций случайного процесса $\mu_{0}(\tau)$, ни разу не достигших границ $y=-\infty, y=h[13]$

$$
F_{0}(h)=\int_{-\infty}^{h} W\left(y, T_{2}\right) d y
$$

Плотность вероятности $W(y, \tau)$ является решением уравнения Фоккера-Планка-Колмогорова (ФПК) $[12,13]$

$$
\frac{\partial W(y, \tau)}{\partial \tau}+\frac{\partial}{\partial y}\left[k_{1} W(y, \tau)\right]-\frac{1}{2} \frac{\partial^{2}}{\partial y^{2}}\left[k_{2} W(y, \tau)\right]=0,
$$

с коэффициентами (16) $k_{1}=k_{10}, k_{2}=k_{20}$, при начальном условии

$$
W\left(y, T_{1}\right)=\frac{1}{\sqrt{2 \pi \lambda_{0}}} \exp \left(-\frac{\left(y+\lambda_{0} / 2\right)^{2}}{2 \lambda_{0}}\right)
$$

и граничных условиях

$$
W(-\infty, \tau)=W(h, \tau)=0 .
$$

Решая уравнение ФПК (19) методом отражения с переменой знака [13], подставляя найденное решение в (18), а затем (18) в (17), получаем выражение для вероятности ложной тревоги

$$
\begin{aligned}
\alpha=\alpha & (h, z)=1-\frac{1}{\sqrt{2 \pi \lambda_{0}}} \int_{0}^{\infty} \exp \left(-\frac{\left(h-\xi+\lambda_{0} / 2\right)^{2}}{2 \lambda_{0}}\right) \\
& \times\left[\Phi \left(\frac{1}{2} \sqrt{\frac{\psi_{0}(k-1)}{k}}+\xi \sqrt{\left.\frac{k}{\psi_{0}(k-1)}\right)}\right.\right. \\
& \left.-\exp (-\xi) \Phi\left(\frac{1}{2} \sqrt{\frac{\psi_{0}(k-1)}{k}}-\xi \sqrt{\frac{k}{\psi_{0}(k-1)}}\right)\right] d \xi,
\end{aligned}
$$

где $k=T_{2} / T_{1}-$ динамический диапазон возможных значений длительности, а

$$
\Phi(x)=\frac{1}{\sqrt{2 \pi}} \int_{-\infty}^{x} \exp \left(-t^{2} / 2\right) d t
$$

- интеграл вероятности.

Далее найдем приближенное выражение для условной вероятности пропуска сигнала, справедливое при достаточно больших ОСШ. Как известно [6,7], при увеличении ОСШ положение максимума логарифма ФОП (7) сходится в среднеквадратическом к положению максимума его математического ожидания $\tau_{s}=\arg \sup S_{1}(\tau)$. Поэтому исследуем решающую статистику $L_{1}(\tau)$ в окрестности $\tau_{s}$. Ограничимся далее рассмотрением таких сочетаний ожидаемых и истинных значений амплитуды и начальной фазы, при которых положение максимума математического ожидания (10) совпадает с истинным значением неизвестной длительности, так что $\tau_{s}=\tau_{0}$. Разложим функции (10) и (12) в ряды Тейлора по $\tau$ в окрестности $\tau_{0}$ и ограничимся членами первого порядка малости, получим асимптотические выражения для математического ожидания и корреляционной функции при наличии сигнала

$$
\begin{gathered}
S_{1}(\tau) \approx \frac{\lambda_{1}}{2}+\frac{\tau-\tau_{0}}{2 T_{2}} \begin{cases}\psi_{1}, & \tau \leq \tau_{0}, \\
-\psi_{2}, & \tau>\tau_{0},\end{cases} \\
K_{q 1}\left(\tau_{1}, \tau_{2}\right) \approx \lambda_{1}+\psi_{2} \min \left(\tau_{1}-\tau_{0}, \tau_{2}-\tau_{0}\right) / T_{2},
\end{gathered}
$$

где

$$
\begin{gathered}
\lambda_{1}=S_{1}\left(\tau_{0}\right), \quad \psi_{1}=z^{2} \Delta_{a} f^{2}\left(\tau_{0}\right) \cos \left(\omega \tau_{0}-\Delta_{\varphi}-\varphi_{0}\right) \\
\times\left[2 \cos \left(\varphi_{0}-\omega \tau_{0}\right)-\Delta_{a} \cos \left(\omega \tau_{0}-\Delta_{\varphi}-\varphi_{0}\right)\right], \\
\psi_{2}=\Delta_{a}^{2} z^{2} f^{2}\left(\tau_{0}\right) \cos ^{2}\left(\omega \tau_{0}-\Delta_{\varphi}-\varphi_{0}\right) .
\end{gathered}
$$

Будем аппроксимировать логарифм ФОП $L_{1}(\tau)$ гауссовским случайным процессом $\mu_{1}(\tau)$ с математическим ожиданием (21) и корреляционной функцией (22). Такая аппроксимация имеет смысл для 


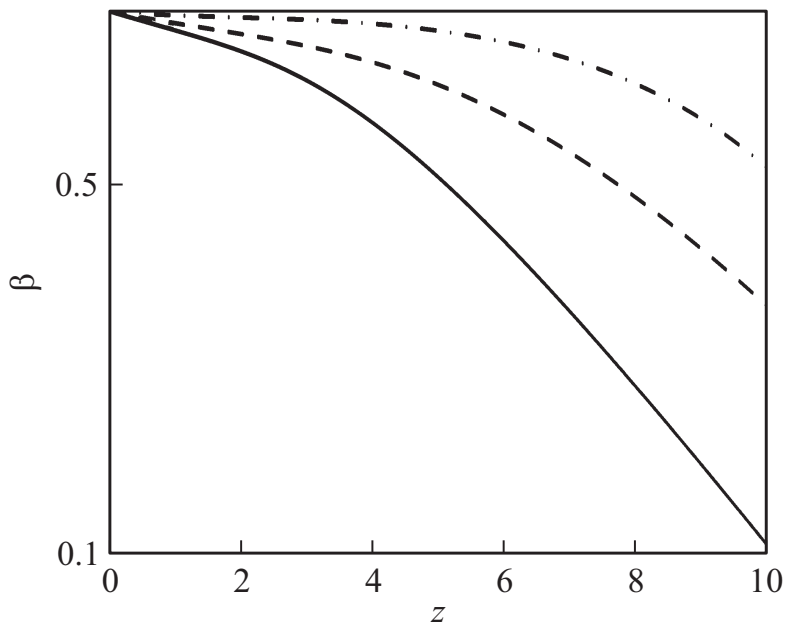

Рис. 3. Зависимость вероятности пропуска сигнала от ОСШ при различных уровнях вероятности ложной тревоги.

всех $\tau>\tau_{d}=\tau_{0}-T_{2} \lambda_{1} / \psi_{2}, \quad$ при которых дисперсия случайного процесса $\mu_{1}(\tau)$ неотрицательна, т.е. $K_{q 1}(\tau, \tau) \approx \lambda_{1}+\psi_{2}\left(\tau-\tau_{0}\right) / T_{2} \geq 0$. При использовании аппроксимации $\mu_{1}(\tau)$ будем считать, что длительность принимает значения из априорного интервала $\left[T_{d}, T_{2}\right]$, где $T_{d}=\max \left(\tau_{d}, T_{1}\right)$. Используя выражения $(21),(22)$ и теорему Дуба $[12,13]$, можно показать, что решающая статистика $\mu_{1}(\tau)$ является гауссовским марковским процессом с коэффициентом сноса $k_{11}$ и коэффициентом диффузии $k_{21}[12,13]$

$$
k_{11}=\frac{1}{2 T_{2}}\left\{\begin{array}{ll}
\psi_{1}, & T_{d} \leq \tau \leq \tau_{0}, \\
-\psi_{2}, & \tau_{0}<\tau \leq T_{2},
\end{array} \quad k_{21}=\frac{\psi_{2}}{T_{2}} .\right.
$$

Вероятность пропуска сигнала по определению равна

$$
\beta=F_{1}(h)=P\left\{\mu_{1}(\tau)<h, \quad \tau \in\left[T_{d}, T_{2}\right]\right\}
$$

- вероятности недостижения границ $y=-\infty$ и $y=h$ марковским случайным процессом $\mu_{1}(\tau)$ на интервале $\tau \in\left[T_{d}, T_{2}\right]$. Искомую вероятность (24) можно выразить через плотность вероятности $W(y, \tau)$ реализаций случайного процесса $\mu_{1}(\tau)$, ни разу не достигших границ $y=-\infty, y=h[13]$

$$
F_{1}(h)=\int_{-\infty}^{h} W\left(y, T_{2}\right) d y .
$$

Функция $W(y, \tau)$ является решением уравнения ФПК (19) с коэффициентами (23) при начальном условии

$$
W\left(y, T_{d}\right)=\frac{1}{\sqrt{2 \pi \sigma^{2}}} \exp \left(-\frac{(y-m)^{2}}{2 \sigma^{2}}\right)
$$

и граничных условиях

$$
W(-\infty, \tau)=W(h, \tau)=0,
$$

где

$$
\sigma^{2}=\lambda_{1}+\psi_{2}\left(T_{d}-\tau_{0}\right) / T_{2}, \quad m=\lambda_{1} / 2+\psi_{1}\left(T_{d}-\tau_{0}\right) / 2 T_{2} .
$$

Решая уравнение ФПК (19) методом отражения с переменой знака [13], подставляя найденное решение в (25), а затем (25) в (24), получаем выражение для вероятности пропуска сигнала

$$
\begin{aligned}
\beta(h, z) & =\frac{\exp \left[-\psi_{1}^{2}\left(k_{d}(1+k)-2 k\right) / 16 \psi_{2} k k_{d}\right]}{\sqrt{\pi \psi_{2}\left(k_{d}(1+k)-2 k\right) / k k_{d}}} \\
& \times \int_{0}^{\infty} \int_{0}^{\infty} W\left(h-\xi, T_{d}\right) \exp \left(\frac{\psi_{1}}{2 \psi_{2}}\left(\xi-\xi_{1}\right)\right) \\
& \times\left\{\Phi \left(\frac{1}{2} \sqrt{\left.\frac{\psi_{2}(k-1)}{2 k}+\xi_{1} \sqrt{\frac{2 k}{\psi_{2}(k-1)}}\right)}\right.\right. \\
& \left.-\exp \left(-\xi_{1}\right) \Phi\left(\frac{1}{2} \sqrt{\frac{\psi_{2}(k-1)}{2 k}}-\xi_{1} \sqrt{\frac{2 k}{\psi_{2}(k-1)}}\right)\right\} \\
& \times\left\{\exp \left(-\frac{\left(\xi-\xi_{1}\right)^{2} k k_{d}}{\psi_{2}\left(k_{d}(1+k)-2 k\right)}\right)\right. \\
& \left.-\exp \left(-\frac{\left(\xi+\xi_{1}\right)^{2} k k_{d}}{\psi_{2}\left(k_{d}(1+k)-2 k\right)}\right)\right\} d \xi_{1},
\end{aligned}
$$

где $k_{d}=\frac{T_{2}}{T_{d}}$.

В качестве примера проанализируем КП алгоритм обнаружения СШП КРС, форма модулирующей функции которого определяется выражением $f(t)=\exp \left(-v t / T_{2}\right)$, где $v$ характеризует скорость убывания модулирующей функции. Введем в рассмотрение величину $\kappa=\omega \tau_{0} / 2 \pi$, численно равную количеству периодов гармонической несущей, укладывающихся на длительности сигнала $\tau_{0}$. Аналогично [4,5] будем называть $\kappa$ параметром узкополосности. При $\kappa \rightarrow \infty$ сигнал (1) является узкополосным. На рис. 3 показаны зависимости вероятности пропуска сигнала (26) от ОСШ (15) при различных уровнях вероятности ложной тревоги (20): сплошная кривая соответствует уровню $\alpha=0.1$, штриховая $-\alpha=0.01$, штрихпунктирная $\alpha=0.001$. При расчете кривых на рис. 3 предполагалось, что начальная фаза принятого сигнала $\varphi_{0}=0, v=2, k=4, \kappa=0.3$, и отсутствуют расстройки амплитуды $\Delta_{a}=1$ и начальной фазы $\Delta_{\varphi}=0$. Зависимости, показанные на рис. 3 , характеризуют эффективность обнаружения при отсутствии расстроек амплитуды и начальной фазы. При этом КП алгоритм обнаружения совпадает с МП алгоритмом при a priori известных амплитуде и начальной фазе.

Влияние априорного незнания амплитуды и начальной фазы на эффективность обнаружения можно количественно охарактеризовать величиной

$$
\chi=\frac{\beta\left(h^{\prime}, z, \Delta_{a}, \Delta_{\varphi}\right)}{\beta\left(h^{\prime}, z, \Delta_{a}=1, \Delta_{\varphi}=0\right)},
$$

которая представляет собой отношение вероятностей пропуска сигнала с неизвестными амплитудой, начальной фазой и длительностью при наличии и отсутствии 


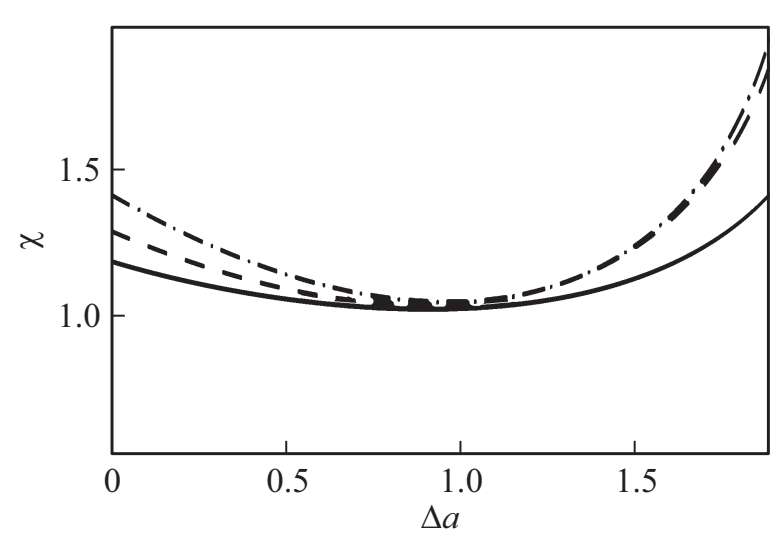

Рис. 4. Зависимость проигрыша в эффективности обнаружения СШП КРС от расстроек амплитуды.

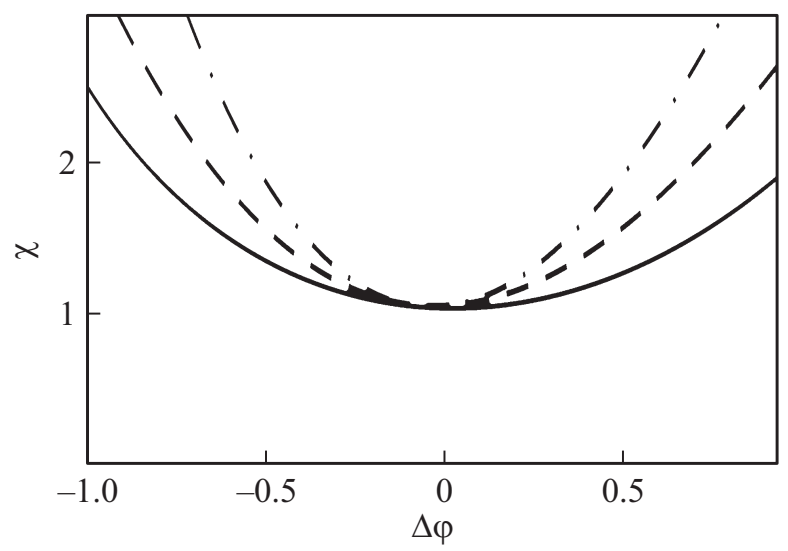

Рис. 5. Зависимость проигрыша в эффективности обнаружения СШП КРС от расстроек начальной фазы.

расстроек по амплитуде и фазе. В (27) $h^{\prime}-$ порог, найденный из решения уравнения $\alpha\left(h^{\prime}, z\right)=p$. На рис. 4 и 5 показаны зависимости проигрыша (27) в эффективности обнаружения СШП КРС с неизвестной длительностью от расстроек амплитуды $\Delta_{a}$ и начальной фазы $\Delta_{\varphi}$ соответственно при различных значениях ОСШ и $p=0.1$. Сплошные кривые на рис. 4,5 соответствуют ОСШ $z=3$, штриховые $-z=5$, а штрихпунктирные $z=7$. При расчете кривых предполагалось, что начальная фаза принятого сигнала $\varphi_{0}=0, v=2, k=4$, $\kappa=0.3$.

Таким образом, априорное незнание амплитуды, начальной фазы и длительности СШП КРС приводит к росту вероятностей ошибок в несколько раз. Следует заметить, что эффективность обнаружения существенно зависит от параметра узкополосности $\kappa$. Это связано с тем, что асимптотически при больших ОСШ вероятности ошибок не зависят от формы сигнала, а определяются лишь величиной скачка его заднего фронта $f\left(\tau_{0}\right)$, который в свою очередь определяется величиной $\kappa$. Приведенные результаты позволяют количественно охарактеризовать влияние априорного незнания параметров
СШП КРС на эффективность обнаружения, а также вычислить допустимые расстройки амплитуды и начальной фазы СШП КРС, исходя из приемлемых вероятностей ошибок ложной тревоги и пропуска сигнала.

Авторы выражают признательность ушедшему из жизни на этапе верстки статьи соавтору - заслуженному деятелю науки РФ, профессору, доктору технических наук Трифонову Андрею Павловичу.

\section{Список литературы}

[1] Радзиевский В.Г., Трифонов П.А. Обработка сверхширокополосных сигналов на фоне помех. М.: Радиотехника, 2009. $288 \mathrm{c}$.

[2] Arslan H., Chen Z.N., Benedetto M.G. Ultra Wideband Wireless Communication. New Jersey: John Wiley \& Sons, 2006. $520 \mathrm{p}$.

[3] Nekoogar F., Dowla F. Ultra-Wideband Radio Frequency Identification Systems. NY: Springer, 2011. 160 p.

[4] Трибонов А.П., Руднев П.Е. // Известия вузов. Радиофизика. 2009. Т. 52. Вып. 9. С. 749-760.

[5] Трифонов А.П., Руднев П.Е. // Известия вузов. Радиофизика. 2011. Т. 54. Вып. 6. С. 458-473.

[6] Акимов П.С., Бакут П.А., Богданович В.А. Теория обнаружения сигналов. М.: Радио и связь, 1984. 440 с.

[7] Тихонов В.И. Оптимальный прием сигналов. М.: Радио и связь, 1983. $320 \mathrm{c.}$

[8] Трибонов А.П., Корчагин Ю.Э., Трифонов М.В. // Известия вузов. Радиофизика. 2015. Т. 58. Вып. 5. С. 401-414.

[9] Куликов Е.И., Трибонов А.П. Оценка параметров сигналов на фоне помех. М.: Сов. радио, 1978. 296 с.

[10] Трифонов А.П., Корчагин Ю.Э., Титов К.Д. // Радиотехника. 2016. Вып. 6. С. 99-105.

[11] Трифонов А.П., Шинаков Ю.С. Совместное различение сигналов и оценка их параметров на фоне помех. М.: Радио и связь, 1986. 264 с.

[12] Doob J.L. Stochastic processes. N.Y.: John Wiley, 1953. 605 c.

[13] Тихонов В.И., Миронов М.А. Марковские процессы. М.: Радио и связь, 1977. 488 с. 